\title{
ON SOME SUBCLASSES OF STRONGLY STARLIKE ANALYTIC FUNCTIONS
}

\section{EL MOCTAR OULD BEIBA*}

El Moctar Ould Beiba, Department of Mathematics and Computer Sciences, Faculty of Sciences and Techniques, University of Nouakchott Al Aasriya, P.O. Box 5026, Nouakchott, Mauritania

${ }^{*}$ Corresponding author: elbeiba@yahoo.fr

AbStRact. The aim of the present article is to investigate a family of univalent analytic functions on the unit disc $\mathbb{D}$ defined for $M \geq 1$ by

$$
\Re\left(\frac{z f^{\prime}(z)}{f(z)}\right)>0,\left|\left(\frac{z f^{\prime}(z)}{f(z)}\right)^{2}-M\right|<M, z \in \mathbb{D} .
$$

Some proprieties, radius of convexity and coefficient bounds are obtained for classes in this family.

\section{INTRODUCTION}

Let $\mathcal{A}$ be the set of analytic function on the unit disc $\mathbb{D}$ with the normalization $f(0)=f^{\prime}(0)-1=0$. $f \in \mathcal{A}$ if $f$ is of the form

$$
f(z)=z+\sum_{n=2}^{+\infty} a_{n} z^{n}, \quad z \in \mathbb{D}
$$

$\mathcal{S}$ denotes the subclass of $\mathcal{A}$ of univalent functions. A function $f \in \mathcal{S}$ is said to be strongly starlike of order $\alpha, 0<\alpha \leq 1$, if it satisfies the condition

$$
\left|\operatorname{Arg} \frac{z f^{\prime}(z)}{f(z)}\right|<\frac{\alpha \pi}{2}, \forall z \in \mathbb{D}
$$

Received April 12 $2^{\text {th }}, 2020$; accepted May $8^{\text {th }}$, 2020; published July 28 ${ }^{\text {th }}, 2020$.

2010 Mathematics Subject Classification. 30C45.

Key words and phrases. strongly Starlike Functions; Subordination; Radius of Convexity; Coefficient Bounds.

(C)2020 Authors retain the copyrights of their papers, and all open access articles are distributed under the terms of the Creative Commons Attribution License. 
This class is denoted by $\mathcal{S S}^{*}(\alpha)$ and was first introduced by D. A. Brannan and W. E. Kirwan [1] and independently by J. Stankiewicz [9].

$\mathcal{S S}^{*}(1)$ is the well known class $\mathcal{S}^{*}$ of starlike functions. Recall that a function $f \in \mathcal{S}$ belongs to $\mathcal{S}^{*}$ if the image of $\mathbb{D}$ under $f$ is a starlike set with respect to the origin or, equivalently, if

$$
\Re\left(\frac{z f^{\prime}(z)}{f(z)}\right)>0, z \in \mathbb{D} .
$$

A function $f \in \mathcal{S}$ belongs to $\mathcal{S S}^{*}(\alpha)$ if the image of $\mathbb{D}$ under $\frac{z f^{\prime}(z)}{f(z)}$ lies in the angular sector

$$
\Omega_{\alpha}=\left\{z \in \mathbb{C},|\operatorname{Arg} z|<\frac{\alpha \pi}{2}\right\}
$$

Let $\mathcal{B}$ denotes the set of Schwarz functions, i.e. $\omega \in \mathcal{B}$ if and only $\omega$ is analytic in $\mathbb{D}, \omega(0)=0$ and $|\omega(z)|<1$ for $z \in \mathbb{D}$. Given two functions $f$ and $g$ analytic in $\mathbb{D}$, we say that $f$ is subordinate to $g$ and we write $f \prec g$ if there exists $\omega \in \mathcal{B}$ such that $f=g \circ \omega$ in $\mathbb{D}$.

If $g$ is univalent on $\mathbb{D}, f \prec g$ is equivalent to $f(0)=g(0)$ and $f(\mathbb{D}) \subset g(\mathbb{D})$.

We obtain from the Schwarz lemma that if $f \prec g$ then $\left|f^{\prime}(0)\right| \leq\left|g^{\prime}(0)\right|$. As a consequence of this statement, we have

$$
f, g \in \mathcal{A}, \frac{f(z)}{z} \prec \frac{g(z)}{z} \Longrightarrow\left|a_{2}\right| \leq\left|b_{2}\right|,
$$

where $a_{2}$ and $b_{2}$ are respectively the second coefficients of $f$ and $g$.

W. Janowski [2] investigated the subclass

$$
S^{*}(M)=\left\{f \in \mathcal{S}, \frac{z f^{\prime}(z)}{f(z)} \in \mathcal{D}_{M}, \forall z \in \mathbb{D}\right\}
$$

where

$$
\mathcal{D}_{M}=\{w \in \mathbb{C},|w-M|<M\}, M \geq 1
$$

J. Sókol and J. Stankiewicz [8] introduced a subclass of $\mathcal{S S}^{*}\left(\frac{1}{2}\right)$, namely, the class $\mathcal{S}_{L}^{*}$ defined by

$$
\mathcal{S}_{L}^{*}=\left\{f \in \mathcal{S}, \frac{z f^{\prime}(z)}{f(z)} \in \mathcal{L}_{1}, \forall z \in \mathbb{D}\right\}
$$

where

$$
\mathcal{L}_{1}=\left\{w \in \mathbb{C}, \Re w>0,\left|w^{2}-1\right|<1\right\} .
$$

$\mathcal{L}_{1}$ is the interior of the right half of the Bernoulli's lemniscate $\left|w^{2}-1\right|=1$.

In the present paper we are interested to the family of subclass of $\mathcal{S}$

$$
\mathcal{S}_{L}^{*}(M)=\left\{f \in \mathcal{S}, \frac{z f^{\prime}(z)}{f(z)} \in \mathcal{L}_{M}, \forall z \in \mathbb{D}\right\}, \quad M \geq 1,
$$


where

$$
\mathcal{L}_{M}=\left\{w \in \mathbb{C}, \Re w>0,\left|w^{2}-M\right|<M\right\}
$$

is the interior of the right half of the Cassini's oval $\left|w^{2}-M\right|=M$. For the particular case $M=1, \mathcal{S}_{L}^{*}(1)$ stands for the class $\mathcal{S}_{L}^{*}$ introduced by J. Sókol and J. Stankiewicz [8]. Since $\mathcal{L}_{M} \subset \Omega\left(\frac{1}{2}\right)$, all functions in $\mathcal{S}_{L}^{*}(M)$ are strongly starlike of order $\frac{1}{2}$.

Note that all classes above correspond to particular cases of the classes of $\mathcal{S}^{*}(\varphi)$ introduced by W. Ma and D. Minda [3],

$$
\mathcal{S}^{*}(\varphi)=\left\{f \in \mathcal{A}, \quad \frac{z f^{\prime}(z)}{f(z)} \prec \varphi\right\} .
$$

where $\varphi$ is Analytic univalent function with real positive part in the unit disc $\mathbb{D}, \varphi(\mathbb{D})$ is symmetric with respect to the real axis and starlike with respect to $\varphi(0)=1$ and $\varphi^{\prime}(0)>0$.

Let $m=1-\frac{1}{M}$ and $\varphi_{m}$ be the function

$$
\varphi_{m}(z)=\sqrt{\frac{1+z}{1-m z}}, \quad z \in \mathbb{D}
$$

where the branch of the square root is chosen so that $\varphi_{m}(0)=1$. We have

$$
\mathcal{S}_{L}^{*}(M)=\mathcal{S}^{*}\left(\varphi_{m}\right)=\left\{f \in \mathcal{A}, \frac{z f^{\prime}(z)}{f(z)} \prec \varphi_{m}\right\}
$$

Observe that $\mathcal{S}_{L}^{*}$ corresponds to $m=0$ so that $\mathcal{S}_{L}^{*}=\mathcal{S}^{*}(\sqrt{1+z})$.

\section{Some PROperties of THE CLASS $\mathcal{S}_{L}^{*}(M)$}

Let $P$ the class of analytic functions $p$ in $\mathbb{D}$ with $p(0)=1$ and $\Re p(z)>0$ in $\mathbb{D}$. For $M \geq 1$, let

$$
P_{\mathcal{L}}(M)=\left\{p \in P,\left|p^{2}(z)-M\right|<M, z \in \mathbb{D}\right\} .
$$

It is easy to see that $P_{\mathcal{L}}\left(M_{1}\right) \subset P_{\mathcal{L}}\left(M_{2}\right)$ for $M_{1} \leq M_{2}$.

Remark 2.1. A function $f \in \mathcal{A}$ belongs to $\mathcal{S}_{L}^{*}(M)$ if and only if there exists $p \in P_{\mathcal{L}}(M)$ such that

$$
\frac{z f^{\prime}(z)}{f(z)}=p(z), \quad z \in \mathbb{D} \text {. }
$$

Theorem 2.1. A function $f$ belongs to $\mathcal{S}_{L}^{*}(M)$ if and only if there exists $p \in P_{\mathcal{L}}(M)$ such that

$$
f(z)=z \exp \int_{0}^{z} \frac{p(\xi)-1}{\xi} d \xi .
$$

Proof. (2.1) is an immediate consequence of the Remark 2.1 
Let $f_{m} \in \mathcal{A}$ be the unique function such that

$$
\frac{z f_{m}^{\prime}(z)}{f_{m}(z)}=\varphi_{m}(z), \quad z \in \mathbb{D}
$$

with $m=1-\frac{1}{M} \cdot f_{m}$ belongs to $\mathcal{S}_{L}^{*}(M)$ and we have

$$
f_{m}(z)=z \exp \int_{0}^{z} \frac{\varphi_{m}(\xi)-1}{\xi} d \xi .
$$

Evaluating the integral in (2.3), we get

$$
f_{m}(z)=\frac{4 z \exp \int_{1}^{\varphi_{m}(z)} H_{m}(t) d t}{\left(\varphi_{m}(z)+1\right)^{2}}, z \in \mathbb{D},
$$

where

$$
H_{m}(t)=\frac{2 m t+2}{m t^{2}+1}, \quad m=1-\frac{1}{M}
$$

For $M=1, H_{0}$ is the constant function $\mathrm{H}(\mathrm{t})=2$ and we have

$$
f_{0}(z)=\frac{4 z \exp (2 \sqrt{1+z}-2)}{(\sqrt{1+z}+1)^{2}} \text { for } z \in \mathbb{D} .
$$

$f_{0}$ is extremal function for problems in the class $\mathcal{S}_{\mathcal{L}}^{*}$ (see [8]).

It is easy to see that

$$
f_{m}(z)=z+\frac{m+1}{2} z^{2}+\frac{(m+1)(5 m+1)}{16} z^{3}+\frac{(m+1)\left(21 m^{2}+6 m+1\right)}{96} z^{4}+\ldots
$$

We need the following result by St. Ruscheweyh [5]

Lemma 2.1. [ [5], Theorem 1] Let $G$ be a convex conformal mapping of $\mathbb{D}, G(0)=1$, and let

$$
F(z)=z \exp \int_{0}^{z} \frac{G(\xi)-1}{\xi} d \xi .
$$

Let $f \in \mathcal{A}$. Then we have

$$
\frac{z f^{\prime}(z)}{f(z)} \prec G
$$

if and only if for all $|s| \leq 1,|t| \leq 1$

$$
\frac{t f(s z)}{s f(t z)} \prec \frac{t F(s z)}{s F(t z)}
$$

Theorem 2.2. If $f$ belongs to $\mathcal{S}_{L}^{*}(M)$ then

$$
\frac{f(z)}{z} \prec \frac{f_{m}(z)}{z} .
$$

Proof. From (1.5), we obtain by applying Lemma 2.1 to the convex univalent function $G=\varphi_{m}$,

$$
\frac{t f(z)}{f(t z)} \prec \frac{t f_{m}(z)}{f_{m}(t z)} .
$$

Letting $t \longrightarrow 0$, we obtain the desired conclusion. 
Corollary 2.1. Let $f$ belongs to $\mathcal{S}_{L}^{*}(M)$ and $|z|=r<1$, then

$$
-f_{m}(-r) \leq|f(z)| \leq f_{m}(r)
$$

$$
f_{m}^{\prime}(-r) \leq\left|f^{\prime}(z)\right| \leq f_{m}^{\prime}(r)
$$

Proof. (2.7) follows from (2.6). Now If $M \geq 1$ we have $0 \leq m<1$. Thus for $0 \leq r<1$

$$
\min _{|z|=r}\left|\varphi_{m}(z)\right|=\varphi_{m}(-r), \max _{|z|=r}\left|\varphi_{m}(z)\right|=\varphi_{m}(r)
$$

From (2.6) and (2.9) we get (2.8) by applying Theorem 2 ( [3], p. 162).

\section{RAdius OF CONVEXITY FOR THE CLASS $\mathcal{S}_{L}^{*}(M)$}

In the sequel $m=1-\frac{1}{M}$.

For $M \geq 1$, let $\mathcal{P}(M)$ be the family of analytic functions $P$ in $\mathbb{D}$ satisfying

$$
\mathrm{P}(0)=1, \quad|\mathrm{P}(\mathrm{z})-\mathrm{M}|<\mathrm{M}, \text { for } \mathrm{z} \in \mathbb{D} \text {. }
$$

We have

$$
f \in \mathcal{S}_{L}^{*}(M) \Longleftrightarrow \exists P \in \mathcal{P}(M) / \frac{z f^{\prime}(z)}{f(z)}=\sqrt{P} .
$$

We need the two following lemmas by Janowski [2]:

Lemma 3.1. [ [2], Theorem 1] For every $P(z) \in \mathcal{P}(M)$ and $|z|=r, 0<r<1$, we have

$$
\inf _{P \in \mathcal{P}(M)} \Re P(z)=\frac{1-r}{1+m r} .
$$

The infimum is attained by

$$
P(z)=\frac{1-\epsilon z}{1+\epsilon m z}, \quad|\epsilon|=1
$$

Lemma 3.2. (Theorem 2, [2]) For every $P(z) \in \mathcal{P}(M)$ and $|z|=r, 0<r<1$, we have

$$
\inf _{P \in \mathcal{P}(M)} \Re \frac{z P^{\prime}(z)}{P(z)}=-\frac{(1+m) r}{(1-r)(1+m r)} .
$$

The infimum is attained by

$$
P(z)=\frac{1-\epsilon z}{1+\epsilon m z}, \quad|\epsilon|=1 .
$$

Theorem 3.1. The radius of convexity of the class $\mathcal{S}_{L}^{*}(M)$ is is the unique root in $(0,1)$ of the equation

$$
4(1+m r)(1-r)^{3}-(1+m)^{2} r^{2}=0 .
$$


Proof. Let $f \in \mathcal{S}_{L}^{*}(M)$. From (3.2), there exists $P \in \mathcal{P}(M)$ such that

$$
\frac{z f^{\prime}(z)}{f(z)}=\sqrt{P(z)}, \quad z \in \mathbb{D}
$$

(3.8) can be written

$$
z f^{\prime}(z)=f(z) \sqrt{P(z)}
$$

which gives

$$
1+\frac{z f^{\prime \prime}(z)}{f^{\prime}(z)}=\frac{z\left(z f^{\prime}\right)^{\prime}(z)}{z f^{\prime}(z)}=\sqrt{P(z)}+\frac{1}{2} \frac{z P^{\prime}(z)}{P(z)} .
$$

This yields for $|z|=r, \quad 0<r<$,

$$
\Re\left(1+\frac{z f^{\prime \prime}(z)}{f^{\prime}(z)}\right) \geq \inf _{P \in \mathcal{P}(M)} \Re \sqrt{P(z)}+\frac{1}{2} \inf _{P \in \mathcal{P}(M)} \Re \frac{z P^{\prime}(z)}{P(z)} .
$$

Replacing (3.3) and(3.5 in (3.9), we obtain

$$
\Re\left(1+\frac{z f^{\prime \prime}(z)}{f^{\prime}(z)}\right) \geq \sqrt{\frac{1-r}{1+m r}}-\frac{1}{2} \frac{(1+m) r}{(1-r)(1+m r)}
$$

Let $h_{M}$ be defined by

$$
h_{M}=\sqrt{\frac{1-r}{1+m r}}-\frac{1}{2} \frac{(1+m) r}{(1-r)(1+m r)} .
$$

$h_{M}$ is decreasing in the interval $(0,1), h_{M}(0)=1$ and the limit of $h_{M}$ in $1^{-}$is $-\infty$. Let $r_{M-1}$ be the unique solution of $h_{M}(r)=0$ in $(0,1)$, then $f$ is convex on the disc $|z|<r_{M-1}$. On the other hand,

$$
1+\frac{z f_{m}^{\prime \prime}(z)}{f_{m}^{\prime}(z)}=\sqrt{\frac{1+z}{1-m z}}+\frac{1}{2} \frac{(1+m) z}{(1-m z)(1+z)}
$$

vanishes in $z=-r_{M-1}$. Thus $r_{M-1}$ is the best value.

To finish, we observe that the equation $h_{M}(r)=0$ is equivalent in the interval $(0,1)$ to the equation

$$
4(1+m r)(1-r)^{3}-(1+m)^{2} r^{2}=0 .
$$

Remark 3.1. As a consequence of Theorem 3.1 applying for $M=1$, we find Theorem 4 [8] which gives $r_{0}$ the radius of convexity of the class $S_{L}^{*} \cdot r_{0}=\frac{1}{12}(11+\sqrt[3]{\sqrt{44928}-181}-\sqrt[3]{\sqrt{44928}+181}) \approx 0.5679591$

Remark 3.2. As observed above, $\mathcal{S}_{L}^{*}(M)$ increases with $M$. Therefore $r_{M-1}$ decreases when $M$ increases. Let

$$
r_{\infty}=\lim _{M \rightarrow+\infty} r_{M-1}
$$

Substituting in (3.7), we obtain 


$$
\left(1+r_{\infty}\right)\left(1-r_{\infty}\right)^{3}-r_{\infty}^{2}=0
$$

Solving this equation in $(0,1)$, we get

$$
r_{\infty}=\frac{1}{2}(1-\sqrt{2}+\sqrt{\sqrt{8}-1}) \approx 0.46899
$$

We have

$$
r_{\infty} \leq r_{M-1} \leq r_{0}
$$

\section{Coefficient Bounds For $\mathcal{S}_{L}^{*}(M)$}

Theorem 4.1. Let $f(z)=\sum_{n=0}^{\infty} a_{n} z^{n}$ be a function in $\mathcal{S}_{L}^{*}(M)$. Then

for $1 \leq M \leq 2$ we have

$$
\sum_{n=2}^{\infty}\left((1-m) n^{2}-2\right)\left|a_{n}\right|^{2} \leq 1+m
$$

and for $M>2$ we have

$$
\sum_{n \geq \sqrt{\frac{2}{1-m}}}\left((1-m) n^{2}-2\right)\left|a_{n}\right|^{2} \leq 1+m-\sum_{2 \leq k<\sqrt{\frac{2}{1-m}}}\left((1-m) k^{2}-2\right)\left|a_{k}\right|^{2} .
$$

with $m=\frac{M-1}{M}$.

Proof. If $f \in \mathcal{S}_{L}^{*}(M)$ there exists $\omega \in \mathcal{B}$ such that

$$
(1-m \omega(z))\left(z f^{\prime}(z)\right)^{2}-f(z)^{2}=\omega(z) f(z)^{2}, \quad z \in \mathbb{D} .
$$

For $0<r<1$ we have

$$
\begin{aligned}
2 \pi \sum_{n=1}^{\infty}\left|a_{n}\right|^{2} r^{2} & =\int_{0}^{2 \pi}\left|f\left(r e^{i \theta}\right)\right|^{2} d \theta \\
& \geq \int_{0}^{2 \pi}\left|\omega\left(r e^{i \theta}\right)\right|\left|f\left(r e^{i \theta}\right)\right|^{2} d \theta
\end{aligned}
$$

Replacing (4.3) in the right side of (4.5) we obtain

$$
\begin{aligned}
2 \pi \sum_{n=1}^{\infty}\left|a_{n}\right|^{2} r^{2} & \geq \int_{0}^{2 \pi}\left|\left(1-m \omega\left(r e^{i \theta}\right)\right)\left(r e^{i \theta} f^{\prime}\left(r e^{i \theta}\right)\right)^{2}-f\left(r e^{i \theta}\right)^{2}\right| d \theta \\
& \geq \int_{0}^{2 \pi}\left|\left(1-m \omega\left(r e^{i \theta}\right)\right)\left(r e^{i \theta} f^{\prime}\left(r e^{i \theta}\right)\right)^{2}\right| d \theta-\int_{0}^{2 \pi}\left|f\left(r e^{i \theta}\right)^{2}\right| d \theta \\
& \geq(1-m) \int_{0}^{2 \pi}\left|\left(r e^{i \theta} f^{\prime}\left(r e^{i \theta}\right)\right)^{2}\right| d \theta-\int_{0}^{2 \pi}\left|f\left(r e^{i \theta}\right)^{2}\right| d \theta \\
& =2 \pi \sum_{n=1}^{\infty}(1-m) n^{2}\left|a_{n}\right|^{2} r^{2}-2 \pi \sum_{n=1}^{\infty}\left|a_{n}\right|^{2} r^{2}
\end{aligned}
$$


Thus

$$
2 \sum_{n=1}^{\infty}\left|a_{n}\right|^{2} r^{2} \geq \sum_{n=1}^{\infty}(1-m) n^{2}\left|a_{n}\right|^{2} r^{2} .
$$

If we let $r \rightarrow 1^{-}$, we obtain from le last inequality

$$
2 \sum_{n=1}^{\infty}\left|a_{n}\right|^{2} \geq \sum_{n=1}^{\infty}(1-m) n^{2}\left|a_{n}\right|^{2}
$$

which gives,

$$
1+m \geq \sum_{n=2}^{\infty}\left((1-m) n^{2}-2\right)\left|a_{n}\right|^{2} .
$$

Since $(1-m) n^{2}-2 \geq 0$ for all $n \geq 2$ if and only if $1 \leq M \leq 2$ then (4.5) yields (4.1) and (4.2) according to the case $1 \leq M \leq 2$ or $M>2$.

The following corollary is an immediate consequence of (4.2).

Corollary 4.1. Let $f(z)=\sum_{n=0}^{\infty} a_{n} z^{n}$ be a function in $\mathcal{S}_{L}^{*}(M)$. Then

for $1 \leq M \leq 2$ we have

$$
\left|a_{n}\right| \leq \sqrt{\frac{1+m}{(1-m) n^{2}-2}}, \text { for } n \geq 2
$$

and for $M>2$ we have

$$
\left|a_{n}\right| \leq \sqrt{\frac{1+m-\sum_{2 \leq k<\sqrt{\frac{2}{1-m}}}\left((1-m) k^{2}-2\right)\left|a_{k}\right|^{2}}{(1-m) n^{2}-2}} ; \text { for } n \geq \sqrt{\frac{2}{1-m}} .
$$

with $m=\frac{M-1}{M}$.

Remark 4.1. For $M=1,(4.1)$ and (4.6) give respectivly Theorem 1 and Corollary 1 [6].

Theorem 4.2. Let $f(z)=\sum_{n=0}^{\infty} a_{n} z^{n}$ be a function in $\mathcal{S}_{L}^{*}(M)$. Then

(i) $\left|a_{2}\right| \leq \frac{m+1}{2}$, for $0 \leq m \leq 1$;

(ii) $\left|a_{3}\right| \leq \frac{m+1}{4}$, for $0 \leq m \leq \frac{3}{5}$;

(iii) $\left|a_{4}\right| \leq \frac{m+1}{6}$, for $0 \leq m \leq \frac{\sqrt{3}-1}{7}$.

This estimations are sharp.

Proof. If $f \in \mathcal{S}_{L}^{*}(M)$ there exists $\omega(z)=\sum_{n=1}^{\infty} C_{n} z^{n} \in \mathcal{B}$ such that

$$
\left(z f^{\prime}(z)\right)^{2}-f(z)^{2}=\omega(z)\left(m\left(z f^{\prime}(z)\right)^{2}+f(z)^{2}\right), \quad z \in \mathbb{D} .
$$

Let $f(z)^{2}=\sum_{n=2}^{\infty} A_{n} z^{n},\left(z f^{\prime}(z)\right)^{2}=\sum_{n=2}^{\infty} B_{n} z^{n}$. (4.8) becomes

$$
\sum_{n=2}^{\infty}\left(B_{n}-A_{n}\right) z^{n}=\left(\sum_{n=2}^{\infty}\left(m B_{n}+A_{n}\right) z^{n}\right)\left(\sum_{n=1}^{\infty} C_{n} z^{n}\right)
$$


Equating coefficients for $n=2, n=3$ in both sides of (4.9), we obtain

$$
\left(S_{m}\right)\left\{\begin{array}{l}
B_{3}-A_{3}=\left(m B_{2}+A_{2}\right) C_{1} \\
B_{4}-A_{4}=\left(m B_{2}+A_{2}\right) C_{2}+\left(m B_{3}+A_{3}\right) C_{1} \\
B_{5}-A_{5}=\left(m B_{2}+A 2\right) C_{3}+\left(m B_{3}+A_{3}\right) C_{2}+\left(m B_{4}+A_{4}\right) C_{1}
\end{array}\right.
$$

A little calculation yields

$$
A_{2}=a_{1}=1, \quad A_{3}=2 a_{2}, \quad A_{4}=2 a_{3}+a_{2}^{2}, \quad A_{5}=2 a_{4}+2 a_{2} a_{3}
$$

and

$$
B_{2}=a_{1}=1, \quad B_{3}=4 a_{2}, \quad B_{4}=6 a_{3}+4 a_{2}^{2}, \quad B_{5}=8 a_{4}+12 a_{2} a_{3} .
$$

Replacing in $\left(S_{m}\right)$, we obtain

$$
\left\{\begin{array}{l}
\text { (1) } 2 a_{2}=(m+1) C_{1} \\
\text { (2) } 4 a_{3}+3 a_{2}^{2}=(m+1) C_{2}+(4 m+2) a_{2} C_{1} \\
\text { (3) } 6 a_{4}+10 a_{2} a_{3}=(m+1) C_{3}+(2 m+1)(m+1) C_{1} C_{2}+\left((6 m+2) a_{3}+(4 m+1) a_{2}^{2}\right) C_{1}
\end{array}\right.
$$

Since $\left|C_{1}\right| \leq 1$ then (1) implies that $\left|a_{2}\right| \leq \frac{1+m}{2}$. This proves the assertion (i). On the other hand we have from (1) and (2)

$$
a_{3}=\frac{1+m}{4} C_{2}+\frac{(5 m+1)(m+1)}{16} C_{1}^{2}
$$

Thus

$$
\left|a_{3}\right| \leq \frac{1+m}{4}\left(\left|C_{2}\right|+\frac{5 m+1}{4}\left|C_{1}\right|\right) .
$$

It is well known that $\left|C_{2}\right| \leq 1-\left|C_{1}\right|^{2}$. Therefore we obtain

$$
\begin{aligned}
\left|a_{3}\right| & \leq \frac{1+m}{4}\left(1-\left|C_{1}\right|^{2}+\frac{5 m+1}{4}\left|C_{1}\right|\right) \\
& =\frac{1+m}{4}\left(1+\frac{5 m-3}{4}\left|C_{1}\right|\right) .
\end{aligned}
$$

Since $5 m-3 \leq 0$ if and only if $m \leq \frac{3}{5}$ then (4.10) yields the assertion (ii).

Replacing the values of $a_{2}$ and $a_{3}$ in the equation (3), we obtain

$$
\begin{aligned}
a_{4} & =\frac{(m+1)}{6} C_{3}+\frac{(m+1)(9 m+1)}{24} C_{1} C_{2}+\frac{(m+1)\left(21 m^{2}+6 m+1\right)}{96} C_{1}^{3} \\
& =\frac{m+1}{6}\left(C_{3}+\frac{9 m+1}{4} C_{1} C_{2}+\frac{21 m^{2}+6 m+1}{16} C_{1}^{3}\right) .
\end{aligned}
$$

Let $\mu=\frac{9 m+1}{4}$ and $\nu=\frac{21 m^{2}+6 m+1}{16}$. Under the assumption $0 \leq m \leq \frac{\sqrt{3}-1}{7}$, we have $(\mu, \nu) \in D_{1}$ (see [4], p. 127). Therefore by Lemma 2 [4] we obtain

$$
\left|C_{3}+\frac{9 m+1}{4} C_{1} C_{2}+\frac{21 m^{2}+6 m+1}{16} C_{1}^{3}\right| \leq 1
$$


which yields from (4.11) the assertion (iii).

The sharpness of (i) is given by the function $f_{m}$. If we take in (4.8) $\omega(z)=z^{2}$ and $\omega(z)=z^{3}$ successively, we obtain two functions in $\mathcal{S}_{L}^{*}(M)$ :

$$
f_{1, m}(z)=z+\frac{m+1}{4} z^{3}+\ldots \text { and } f_{2, m}(z)=z+\frac{m+1}{6} z^{4}+\ldots
$$

which give respectively the sharpness of estimations (ii) and (iii).

Remark 4.2. The estimation (i) can be obtained directly from (2.6).

Remark 4.3. If we take $m=0$ in Theorem 4.2, we obtain as particular case Theorem 2 [6].

Conflicts of Interest: The author(s) declare that there are no conflicts of interest regarding the publication of this paper.

\section{REFERENCES}

[1] D.A. Brannan, W.E. Kirwan, On Some Classes of Bounded Univalent Functions, Journal of the London Mathematical Society. s2-1 (1969), 431-443.

[2] W. Janowski, Extremal Problems for a Family of Functions with Positive Real Part and for Some related Families, Ann. Polon. Math. 23 (1970), 159-177.

[3] Wancang Ma, David Minda, A Unified Treatment of some Classes of Univalent Functions, Proceeding of the International Conference on Complex Analysis at the Nankai Institute of Mathematics, 1992, 157-169.

[4] Dimitri V. Prokhorov, Jan Szynal, Inverse Coefficients for $(\alpha, \beta)$-convex Functions, Ann. Univ. Mariae Curie-Sklodowska, XXXV (15) (1981), 125-143.

[5] S. Ruscheweyh, A Subordination Theorem for $\Phi$-Like Functions, J. Lond. Math. Soc. s2-13 (1976), 275-280.

[6] Janusz Sókol, Coefficient Estimates in a Class of Strongly Starlike Functions, Kyungpook Math. J. 49 (2009), 349-353.

[7] Janusz Sókol, On Some Subclass of Strongly Starlike Functions, Demonstr. Math. XXXI (1) (1988), 81-86.

[8] J. Sókol, J. Stankiewicz, Radius of Convexity of some Subclass of Strongly Starlike Functions, Folia Sci. Univ. Tech. Resovienis, Math. 19 (1996), 101-105.

[9] J. Stankiewicz, Quelques problèmes extrémaux dans les Classes des Fonctions $\alpha$-angulairement étoilées, Ann. Univ. Mariae Curie-Sklodowska Sect. A, 20 (1966), 59-75. 